\title{
VLA observations of the interacting-wind binary system WR 147
}

\author{
M. Eugenia Contreras and Luis F. Rodríguez \\ Instituto de Astronomía, UNAM, \\ Apdo. Postal 70-264, 04510 México, D.F., México
}

\section{Introduction}

Wolf-Rayet stars are losing mass via their strong winds. Some of them show thermal and non-thermal radio emission and from this group some have companions forming binary systems. It has been suggested that the synchrotron emission arises from the interaction region where the winds of the two stars collide. WR 147 seems to be one of these systems, having a companion and emitting thermal as well as non-thermal radiation. Besides, it has been suggested that it has an anisotropic wind. In the present work we continue studying the WR 147 system by observing it at a radio wavelength of $3.6 \mathrm{~cm}$ in two different epochs. We have looked for evidence to support the idea that WR 147 is a colliding-wind binary system. We have also looked for time variations in the source during the time interval of $\sim 1.4$ years separating our observations.

\section{Discussion}

The thermal main component (WR 147S) shows a flux density increase of $\sim 25 \%$ from 1995 to 1996 . From these data we can adopt 0.1 as the characteristic radius of the emitting region in WR 147S. At a distance of $630 \mathrm{pc}$ (Churchwell et al. 1992) this angular size corresponds to a physical radius of $\sim 63 \mathrm{AU}$. Assuming a terminal velocity of $900 \mathrm{~km} \mathrm{~s}^{-1}$ (Churchwell et al. 1992) for the wind, we find that the dynamical timescale for replacement of the emitting gas is only $\sim 0.33$ years. Thus, the variability in the WR $147 \mathrm{~S}$ flux density could be explained as the result of a change in the wind parameters. The apparent lack of variability in WR $147 \mathrm{~N}$ could be the result of the larger dimensions of this source or that in it we are observing the effects of the winds of WR 147S and the IR companion as they were $\sim 2$ years in the past. From the derived deconvolved angular dimensions of WR $147 \mathrm{~S}$, it is not possible to be certain that the emission comes from an asymmetric wind. The major and minor axes of the fit are both consistent with the value of $0.21 \pm 0$. 02 . Thus, we conclude that for the epoch of our observations, WR 147S appears to be spherically symmetric to first approximation. However, our results show possible faint condensations in the wind that have moved away by $\sim 0 . \prime 2$ from WR $147 \mathrm{~S}$ in a time interval of $\sim 1.4$ years. This fact seems to indicate that we are dealing with an inhomogeneous wind. Finally, from our maps it can be clearly seen that the northern source, WR $147 \mathrm{~N}$, shows an E-W elongation and furthermore, that its shape resembles a shock like those described theoretically by Stevens, Blondin \& Pollock (1992), and Cantó, Raga $\&$ Wilkin (1996). To make a more quantitative comparison between our obser- 
vations and the bow shock models, we have determined a curve that follows the observed shape of WR $147 \mathrm{~N}$. This curve was obtained fixing the ratio of windmomenta between the stars. However, since it is very improbable that we would be looking face-on at the system $(\phi=0, \phi$ defined as the angle between the line joining both stars and the plane of the sky), we have projected our fitted curve onto the plane of the sky following the equations given by Raga et al. (1997). It is important to note that one can obtain a family of curves with pairs of $(\eta, \phi)$ that approximately follow the shock. Since Williams et al. (1997) have determined a value for the wind-momentum ratio $(\eta=0.011)$ based on high resolution infrared and radio observations, we have fixed this value. We have varied then the inclination angle $\phi$ in order to find the best fit to the shape of the shock. In this way, we have derived a range of possible inclination angles of the system $\left(45^{\circ} \pm 15^{\circ}\right)$. It can be shown that $i$, the angle between the normal to the orbit of the binary and the line of sight, is related with $\phi$ by $\sin (i) \geq \sin (\phi)$. Thus, despite the limitations of our present data, this analysis indicates that fitting the shape of the interacting-wind region can provide a lower limit for the inclination angle of the orbit, $i$, generally a poorly known parameter. These results are presented in Contreras \& Rodríguez (1999).

\section{Conclusions}

Our main conclusions are as follows: (a) We find time variations at the $25 \%$ level in the radio flux density of WR $147 \mathrm{~S}$. This variability had been suspected in previous studies but was not proven. We find WR $147 \mathrm{~S}$ to be spherically symmetric. However, our data suggest the possibility of density inhomogenities traveling with the wind; and (b) We confirm that WR $147 \mathrm{~N}$ is a shock produced at the interaction between the winds of the stars forming this binary system, as first proposed by Williams et al. (1997) and Niemela et al. (1998). We have fitted the source shape to a shock model and derived an inclination angle of $45^{\circ} \pm 15^{\circ}$ for the system. This method could potentially be used to estimate the inclination angle of the orbit, $i$, of other interacting-wind binaries.

\section{References}

Churchwell, E.B., Bieging, J.H., van der Hucht, K.A., Williams, P.M., Spoelstra, T.A.Th., Abbott. D.C. 1992, ApJ 393, 329

Cantó, J., Raga, A.C., Wilkin, F.P. 1996, ApJ 469, 729

Contreras, M.E., Rodríguez, L.F. 1999, ApJ 515, 762

Raga, A.C., Cantó, J., Curiel, S., Noriega-Crespo, A., Raymond, J.C. 1997, RevMexAA, 33,157

Stevens, I.R., Blondin, J.M., Pollock, A.M.T. 1992, ApJ 386, 265

Williams, P.M., Dougherty, S.M., Davis, R.J., van der Hucht, K.A., Bode, M.F., Setia Gunawan, D.Y.A. 1997, MNRAS 289, 10 